\title{
TOMOGRAPHIC SAR INVERSION FOR URBAN RECONSTRUCTION
}

\author{
Karima Hadj-Rabah ${ }^{1}$, Faiza Hocine ${ }^{2}$, Assia Kourgli ${ }^{3}$ and Aichouche \\ Belhadj-Aissa ${ }^{4}$ \\ ${ }^{1,2,3,4}$ Department of Telecommunications, University of Sciences and Technology \\ Houari Boumediene (USTHB), Algiers, Algeria
}

\begin{abstract}
Given its efficiency and its robustness in separating the different scatterers present in the same resolution cell, SAR tomography (TomoSAR) has become an important tool for the reflectivity reconstruction of the observed complex structures scenes by exploiting multi-dimensional data. By its principle, TomoSAR reduces geometric distortions especially the layover phenomenon in radar scenes, and thus reconstruct the $3 D$ profile of each azimuth-range pixel. In this paper, we present the results and the comparative study of six tomographic reconstruction methods that we have implemented. The analysis is performed with respect to the separability and location of scatterers by each method, supplemented by the proposal of a quantitative analysis using metrics (accuracy and completeness) to evaluate the robustness of each method. The tests were applied on simulated data with TerraSAR-X sensor parameters.
\end{abstract}

\section{KEYWORDS}

SAR Tomography (TomoSAR), Reconstruction Algorithms, Accuracy\& Completeness

\section{INTRODUCTION}

In the last two decades, SAR tomography (TomoSAR) had a growing interest in remote sensing, particularly after the acquisition of very high resolution (VHR) data acquired by the latest generation of SAR radar sensors such as: TerraSAR-X and CosmoSky-Med. TomoSAR is a new data acquisition method, it exploits a series of SAR images taken with slightly different view angles to reconstruct the 3D profile of the reflectivity function for each azimute-range pixel [1].

The choice of a reconstruction method leading to conclusive results depends, on the one hand, on the nature of the area intended to be analyzed: forested, urban, etc and on the other hand, on the type of data and their characteristics in terms of spatial and temporal resolution. In the case of VHR images, it is common to adopt a deterministic scattering model to model a scene due to the high density of point scatterers with a high signal-to-noise ratio (SNR) present in the latter [2]. However, by its principle, SAR tomography exploits pairs of SAR images acquired simultaneously and / or at different time intervals, so that the temporal and spatial decorrelation problems, the atmospheric delay and the noise of various sources are the main tomographic process limitations and the inaccuracy of reconstructed scenes in terms of amplitude and altimetry.

Several parametric and non-parametric, single- and multi-looking SAR tomographic reconstruction methods have been developed and implemented in literature. Classical Fourier- 
Based focusing and SVD algorithm were the first approaches applied to simulated and real data acquired in the C-band by the ERS sensor [4] [5]. However due to the non-regularity of the acquisitions distribution on the Baseline axis, these two approaches introduce a degradation of the PSF (Point Spread Function) reconstruction according to the elevation in terms of resolution and side lobes. As an alternative to these two classical approaches, CAPON and MUSIC have been proposed in [6] [7] to ensure super-resolution (SR) and good side lobes suppression. Nevertheless, the use of the estimated covariance matrix represents the major disadvantage of CAPON and MUSIC. In [8] [9], Non-Linear Least-Square was proposed despite its high cost, because it provides good accuracy in altitude (Height accuracy).

In addition, the evaluation of the reconstruction methods mentioned above was carried out qualitatively. In [7], the authors used root mean square error (RMSE) to estimate the performance of tomographic reconstruction. Two new metrics have been adapted to TomoSAR in [2] and [10] to define root mean square accuracy $R M S E_{A}$ and root mean square completeness $R M S E_{C}$, the two latter are based on distortion measure by the Euclidean distance in order to measure the error between the estimated targets (estimated scatterers) and the real targets (ground truth targets).

The objective of this work is to present a comparative study between the most widely used reconstruction approaches in the literature, without taking into consideration the sparse property of the radar signals. This study is based on a quantitative evaluation of the treatment results by introducing other criterias to calculate the accuracy and the completeness of the target location in elevation and the restitution of their reflectivities (their amplitudes).

The remainder of the paper is organized in the following way: the next section briefly describes the TomoSAR geometry, in section 3 we will look at the characteristics description of the reconstruction approaches studied, this section will also include a detailed analysis of the evaluation methods suggested by the authors, then the analysis results as well as a comparison between the different approaches will be presented in section 4 , and finally, the paper ends with a conclusion.

\section{TOMOSAR GEOMETRY AND IMAGING MODEL}

Due to side-looking geometry, the projection of 3D objects on the plane (azimuth $x$, range $r$ ) introduces geometric problems in urban and uneven terrain areas causing an ambiguity when interpreting SAR images. To remedy these distortions, TomoSAR makes it possible to separate several scatterers located at different altitudes present in the same resolution cell, by projecting the scatterers responses on an axis perpendicular to the 'azimuth-range' plane. These responses are reconstructed from N SAR images taken at different view angles (see Fig.1). The complex value $g_{n}$ of a coordinate pixel $(\mathrm{x}, \mathrm{r})$ for the $\mathrm{n}^{\text {th }}$ acquisition with $n=1, \ldots, \mathrm{N}$ is a sample of the Fourier transform of the reflectivity function $\gamma(s)$ with respect to the elevation $s$, its expression is [1]:

$$
g_{n}=\int_{\frac{-\Delta_{s}}{2}}^{\frac{\Delta_{s}}{2}} \gamma(s) \exp \left(-j 2 \pi \xi_{n} s\right) d s+w_{n}
$$

With: $\Delta_{s}$ is the elevation interval, $\xi_{n}=-2 b_{n} / \lambda r$ represents the spatial frequency which depends on the sensor position $b_{n}$ on the Baseline axis, $\lambda$ is the wavelength and $w_{n}$ is the noise, the latter follows a Gaussian distribution [11]. 


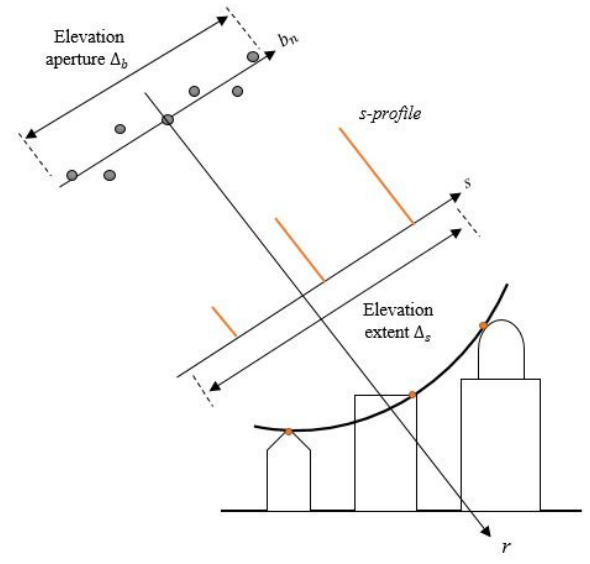

Figure 1. TomoSAR geometry

Equation (1) can be modeled by the following linear system:

$$
g=A Y+W
$$

With: $g$ is the observations vector of length $\mathrm{N}, A$ is the steering vectors matrix of size NxM, and $Y$ is the reflectivity profile uniformly sampled at $s_{m}$ with $m=1, . .$, M. To ensure SR, M must be very large $(M \gg N)$. Therefore, the $s$-profile recovery i.e. the $Y$ profile reconstruction is a spectral estimation problem.

\section{TOMOGRAPHIC RECONSTRUCTION APPROACHES AND EVALUATION METHODS}

\subsection{Reconstruction Approaches}

Tomographic reconstruction approaches can be classified into: non-parametric approaches and parametric approaches, or single-looking and multi-looking approaches. Non-parametric approaches allow the estimation of power spectral density whose statistical properties are unknown in prior, by passing the observed data through a set of bandpass filters in order to estimate the output power, while parametric approaches model the observed data by a few sinusoids in order to estimate their parameters. A better estimation can be offered by the latter only if the suggested model is close to the processed data [12].

Single-looking approaches do not exploit the correlation between the pixel set to be processed and its neighborhood, whereas multi-looking approaches require the covariance matrix estimation from the observations vector $(\widehat{R})$, this ensures a SR in elevation but with a considerable loss of the reconstructed scenes spatial resolution.

The most implemented reconstruction methods in literature are Conventional Beamforming (CBF), Beamforming (BF), Adaptive Beamforming (CAPON), TSVD, MUSIC and Non-linear Least Square (NL-LS), their characteristics description is summarized in Table 1: their equations, their ranking (parametric or non-parametric, single- or multi-looking), their resolution as well as their advantages and disadvantages. 
Table 1. Characteristics of Tomographic reconstruction methods

\begin{tabular}{|c|c|c|c|c|c|}
\hline Method & Parametric & $\begin{array}{l}\text { Multi- } \\
\text { looking }\end{array}$ & Equation & $\begin{array}{l}\text { Elevation } \\
\text { resolution }\end{array}$ & Advantages and Disadvantages \\
\hline $\begin{array}{l}\text { Conventional } \\
\text { Beamforming } \\
\text { (CBF) }\end{array}$ & No & No & $\hat{Y}=A^{H} g$ & Poor & 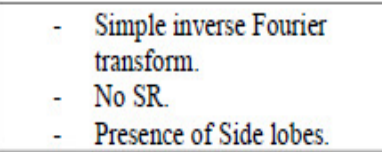 \\
\hline $\begin{array}{l}\text { Beamforming } \\
\text { (BF) }\end{array}$ & No & Yes & $\hat{Y}=\frac{A^{H} \hat{R} A}{N^{2}}$ & $\begin{array}{l}\text { Poor / } \\
\text { Average }\end{array}$ & $\begin{array}{l}\text { Biased estimator when } \\
\text { Number of scatterers is } \\
\text { greater than } 1 .\end{array}$ \\
\hline $\begin{array}{l}\text { Adaptive } \\
\text { Beamforming } \\
\text { (Capon) }\end{array}$ & No & Yes & $\hat{Y}=\frac{\hat{R}^{-1} A}{A^{H} \hat{R}^{-1} A} \cdot g$ & Average & $\begin{array}{l}\text { - Requires a big number of } \\
\text { looks. } \\
\text { - Better scatterers separation } \\
\text { but the amplitude estimation } \\
\text { is biased. }\end{array}$ \\
\hline TSVD & No & No & $\begin{array}{l}\quad \hat{Y}=\left(V \cdot \frac{1}{S}, U^{H}\right) \cdot\left(G_{m, n} \cdot g\right) \\
\text { With: } \\
G_{m, n}=\Delta_{s} \cdot \operatorname{sinc}\left(\Delta_{s}\left(\xi_{m}-\xi_{n}\right)\right) \\
\text { And }[U, S, V]=\operatorname{svd}(A)\end{array}$ & $\begin{array}{l}\text { Poor / } \\
\text { Average }\end{array}$ & $\begin{array}{l}\text { Stable for well-defined } \\
\text { problems (well-defined } \\
\text { truncation rank T). }\end{array}$ \\
\hline MUSIC & Yes & Yes & $\begin{array}{c}\qquad \hat{Y}=\left|\frac{1}{A^{H} G^{H} G A}\right|^{2} \\
\text { With } G \text { the noise subspace. }\end{array}$ & High & $\begin{array}{l}\text { - Better elevation resolution } \\
\text { and weak side lobes. }\end{array}$ \\
\hline $\begin{array}{l}\text { Non-Linear } \\
\text { Least-Square } \\
\text { (NL-LS) }\end{array}$ & Yes & No & $\begin{array}{l}\hat{Y}=\min _{k}\left\{\|g-A Y\|_{2}^{2}\right\} \\
=\left(A^{H} A\right)^{-1} A^{H} g\end{array}$ & $\begin{array}{l}\text { High / Very } \\
\text { High }\end{array}$ & $\begin{array}{l}\text { - Better amplitude estimation } \\
\text { (if }(\xi) \text { is precised). }\end{array}$ \\
\hline
\end{tabular}

\subsection{Evaluation Methods}

Our comparative study is based on a quantitative evaluation that uses two metrics: precision and completeness, recently proposed in [2]. Precision provides a measure that describes how the estimated targets $\hat{P}$ are close to the real targets $P$, its expression is as follows:

$$
A_{\text {dist }}=\frac{1}{N_{p}} \sum_{j=1}^{N_{p}} \min _{k} \operatorname{dist}\left(\hat{P}_{j}, P_{k}\right)
$$

While completeness is a measure of how real targets are modeled by estimated targets, its expression is:

$$
C_{\text {dist }}=\frac{1}{N_{p}^{\prime}} \sum_{j=1}^{N^{\prime} p} \min _{k} \operatorname{dist}\left(\widehat{P}_{k}, P_{j}\right)
$$

With $N_{p}$ and $N_{p}^{\prime}$ are the number of estimated and real targets respectively, dist represents the distortion measure.

We adapted these two measurements to our analysis by estimating the elevation position of each target scatterer as well as its reflectivity (amplitude) separately, therefore, we defined Elevation 
Accuracy, Amplitude Accuracy, Elevation Completeness, and Amplitude Completeness. In addition, in order to obtain more consistent results, we used the Manhattan distance (D1) and the Euclidean distance (D2) to measure the distortion.

\section{RESULTS AND DISCUSSION}

Our study includes three phases, the first one consists of simulating tomographic SAR data, the second step involves the implementation of reconstruction methods, and the last phase is dedicated to evaluating the obtained results.

The targets reconstruction tests, by each algorithm, were performed on a simulated profile along the elevation axis from the TerraSAR-X satellite parameters (see Table 2), assuming that the scatterers number is equal to 3 in one resolution cell (see Fig. 1) with a SNR of $10 \mathrm{~dB}$.

Table 2. TerraSAR-X parameters.

\begin{tabular}{|l|l|}
\hline $\begin{array}{l}\text { Distance from the } \\
\text { scene center [m] }\end{array}$ & 740000 \\
\hline $\boldsymbol{\Delta}_{\boldsymbol{s}}[\mathbf{m}]$ & 269,5 \\
\hline $\boldsymbol{\lambda}[\mathbf{m}]$ & 0,031 \\
\hline $\mathbf{N}$ & 25 \\
\hline
\end{tabular}

A synthesis aperture in elevation was effectuated, by the following with random samples distribution scheme on the Baseline axis, this samples distribution is represented in the graph of Figure 2. We performed an implementation of the tomographic reconstruction methods described in the previous section, the resulting profiles are shown in Figure 3.

The evaluation results of the different reconstructions accuracy are shown in Tables 3, 4 and 5 . The elevation and amplitudes accuracy and completeness were calculated after applying a 3peaks detector on the curves of Figure 3 to compare between them.

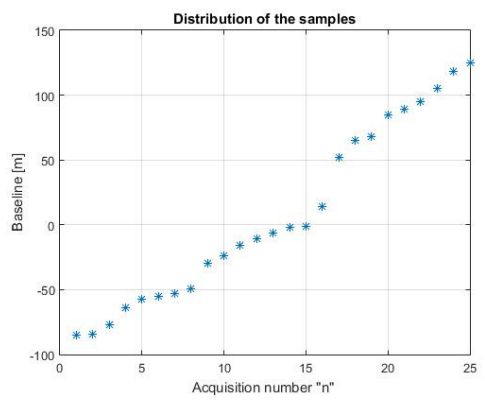

Figure 2. Samples distribution scheme

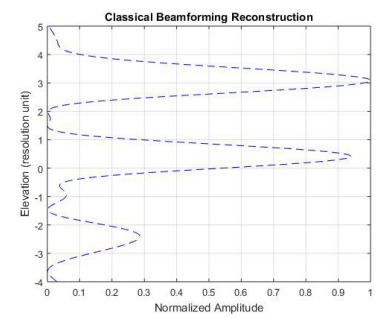

(a)

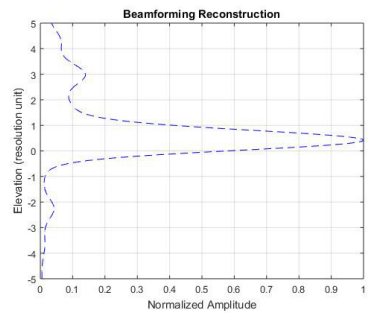

(b)

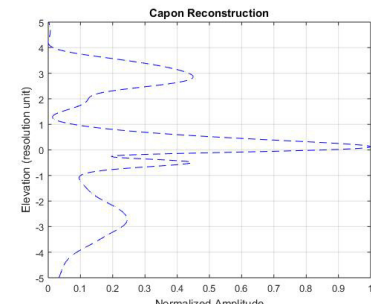

(c) 


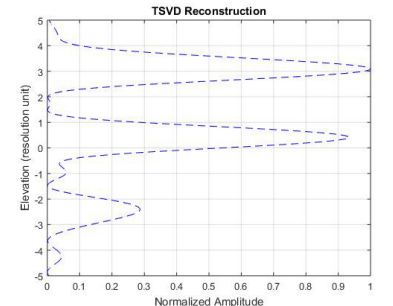

(d)

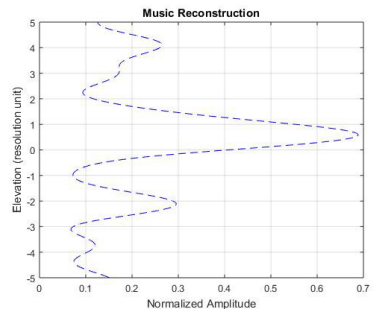

(e)

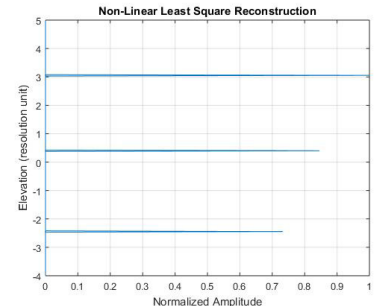

(f)

Figure 3. Reflectivity profile reconstruction by :(a) CBF, (b) BF,(c) CAPON, (d) TSVD, (e) MUSIC and

(f) NL-LSmethod

Table 3. RMSE values of the different reconstruction approaches.

\begin{tabular}{|l|l|l|l|l|l|l|}
\hline Approach & CBF & BF & CAPON & TSVD & MUSIC & NL-LS \\
\hline RMSE & 0.0824 & 0.0714 & 0.0759 & 0.0823 & 0.0657 & 0.0856 \\
\hline
\end{tabular}

Table 4. Elevation Accuracy and completeness of the different reconstruction approaches with a normalization factor of $\left(10^{3}\right)$.

\begin{tabular}{|l|l|l|l|l|l|l|l|}
\hline \multicolumn{2}{|l|}{ Approach } & CBF & BF & CAPON & TSVD & MUSIC & NL-LS \\
\hline \multirow{2}{*}{$\begin{array}{l}\text { Elevation } \\
\text { Accuracy }\end{array}$} & D1 & 0.0027 & 0.0143 & 0.0217 & 0.0023 & 0.0057 & 0.0027 \\
\cline { 2 - 8 } $\begin{array}{l}\text { Elevation } \\
\text { Completeness }\end{array}$ & D1 & 0.0073 & 0.4857 & 0.6897 & 0.0057 & 0.0417 & 0.0073 \\
\cline { 2 - 8 } & D2 & 0.0073 & 4.6457 & 2.1820 & 0.0057 & 0.0417 & 0.0073 \\
\hline
\end{tabular}

Table 5. Amplitude Accuracy and completeness of the different reconstruction approaches.

\begin{tabular}{|l|l|l|l|l|l|l|l|}
\hline \multicolumn{2}{|l|}{ Approach } & CBF & BF & CAPON & TSVD & MUSIC & NL-LS \\
\hline \multirow{2}{*}{$\begin{array}{l}\text { Amplitude } \\
\text { Accuracy }\end{array}$} & D1 & 0.0929 & 0.2581 & 0.0388 & 0.0946 & 0.1925 & 0.0377 \\
\cline { 2 - 8 } D2 & 0.0166 & 0.1001 & 0.0020 & 0.0167 & 0.0504 & 0.0042 \\
\hline $\begin{array}{l}\text { Amplitude } \\
\text { Completeness }\end{array}$ & D1 & 0.1407 & 0.2091 & 0.1058 & 0.1383 & 0.1817 & 0.0778 \\
\cline { 2 - 7 } & D2 & 0.0293 & 0.0668 & 0.0233 & 0.0283 & 0.0449 & 0.0180 \\
\hline
\end{tabular}

The RMSE values of the estimated scatterers by each of the methods described above are presented in Table 3. The latters are close to each other, which leaves us to conclude that the different reconstructions have practically the same precision.

Although the three scatterers were well separated by all the approaches according to the graphs of Figure 3, we note that compared to the 'BF', 'CAPON' and 'MUSIC' approaches, the scatterers localization on the elevation axis is erroneous contrary to the 'CBF', 'TSVD' and 'NL-LS' approaches which effectively allow the reflectivity profile reconstruction with a good estimation of the scatterers location on the elevation axis as well as their amplitudes. We emphasize that the erroneous localization given by 'BF', 'CAPON' and 'MUSIC' is mainly due to the poor covariance matrix estimation of the observations vector caused by the strong noise presence and consequently a non-precise reconstruction. Hence, the evaluation by the RMSE calculation is not the most judicious choice.

To corroborate these graphical results, we calculated the accuracy and completeness using the expressions described in the previous section. The results of these metrics are given in Table 4. The analysis of these measurements shows that the best elevation accuracy and completeness values are those of 'CBF', 'TSVD' and 'NL-LS' methods. Also, from the results of Table 5, we notice that the 'NL-LS' approach has the best amplitude accuracy and completeness values. These results are in conformity with those of Figure 3. We can conclude that the metrics 'Elevation 
Accuracy', 'Amplitude Accuracy', 'Elevation Completeness' and 'Amplitude Completeness' are a tool for performance evaluation of SAR tomographic reconstruction. This observation makes it easier to choose the most appropriate reconstruction approach depending on the surface and / or the sub-surface to be reconstructed. In this sense, if only one scatterer is present in a resolution cell, all the previously described methods reconstruct the target with very good accuracy. However, in the case of multiple scatterers, in an urban area for example it is very important to preserve the spatial resolution in order to be able to observe the urban infrastructures, in this case, the single-looking approaches are privileged. In addition, for medium-resolution applications, it is recommended to apply the TSVD method, as for high resolution applications, the NL-LS approach can provide good performance with a quite important calculation cost.

\section{CONCLUSION}

Tomo-SAR has shown its effectiveness in exploiting very highresolution SAR data for mapping and monitoring urban environments, and in recognizing the ambiguity caused by geometric problems, in particular layover. In this work, we have implemented the most exploited tomographic reconstruction approaches and have analyzed the results of these methods in terms of localization and amplitude of the reconstructed profile interactively, then we have quantified the quality of this reconstruction by metrics expressing accuracy and completeness. We have found a good correlation between the metrics and the reconstructed profiles representation by each method. Therefore, we concluded that accuracy and completeness represent an objective judging way in choosing the most appropriate reconstruction approach.

\section{REFERENCES}

[1] X. X. Zhu\&R.Bamler, (2014) "Superresolving SAR Tomography for Multidimensional Imaging of Urban Areas”, IEEE Signal Processing Magazine.

[2] O. D’Hondt, C. Lopèz-Martinez, S. Guillaso\&O. Hellwich, (2018), "Nonlocal Filtering Applied to 3D Reconstruction of Tomographic SAR Data”, IEEE Transactionson Geoscienceand Remote Sensing, Vol. 56, No. 1, pp 272-285.

[3] L. Linze, P. Lei, Z. XueDong\&L. Hui, (2017), “The Research on Accuracy Evaluation Method of the Tomographic SAR Three-Dimensional Reconstruction of Urban Building Based on Terrestrial Lidar Point Cloud", ISPRS Geospatial week 2017, Wuhan, China.

[4] G. Fornaro, F. Serafino, et al., (2003), "Three-Dimensional Focusing with Multipass SAR Data", IEEE Transactions on Geoscience and Remote Sensing, Vol. 41, No. 3, pp. 507-517.

[5] G.Fornaro, F.Lombardini, et al., (2005), “Three-Dimensional Multipass SAR Focusing: Experiments with Long-Term Spaceborne Data", IEEE Transactions on Geoscience and Remote Sensing, Vol. 43, No. 4, pp702-714.

[6] S. Duque, P. Lopez-Dekker, et al., (2010), "Bistatic SAR Tomographic: Processing and Experimental Results", IGARSS 2010.

[7] F. Lombardini\& F. Cai, (2014), “Temporal decorrelation Robust SAR Tomography”, IEEE Transactions on Geoscience and Remote Sensing, Vol. 52, No. 9, pp 5412-5421.

[8] G.Fornaro\&F. Serafino, (2006), "Imaging of Single and Double Scatterers in Urban Areas via SAR Tomography", IEEE Transactions on Geoscience and Remote Sensing, Vol. 44, No. 12, pp34973505 .

[9] X. X. Zhu\&R. Bamler, (2010), "Very High Resolution Spaceborne SAR Tomography in Urban Environment", IEEE Transactions on Geoscience and Remote Sensing, Vol. 48, No. 12, pp 42964308. 
[10] O. D’Hondt, C. Lopèz-Martinez, S. Guillaso\&O. Hellwich, (2017), "Impact of Non-local Filtering on 3D Reconstruction from Tomographic SAR Data”, IGARSS 2017.

[11] D. Reale, W. Franzè, et al., (2015), "Detection of Single Scatterers in Multilook SAR Tomography”,Joint Urban Remote Sensing Event (JURSE).

[12] P. Stoica\& R. Moses, (2005), Spectral analysis of signals, Pearson Prentice Hall.

\section{AUTHORS}

Karima Hadj-Rabah received the M.Sc. degree in Telecommunication, networks and multimedia from University of Science and technology HouariBoumedienne (USTHB), Algeria, in 2016. She is curently working toward the Ph.D. degree in synthetic aperture radar (SAR) tomography reconstruction using very highresolution data, in laboratory of image processing and radiation, USTHB, under the supervision of Professor AichoucheBelhadj-Aissa.

Faiza Hocine Graduated from the University of Sciences and Technology HouariBoumedienne (USTHB), Algeria. PhD in image processing and remote sensing of the same university, in 2015. Currently, she is researcher in Electronics, image processing, remote sensing at USTHB. Her research interests include satellite image processing, SAR interferometry radar.

AssiaKourgli received her PhD from USTHB University, Algeria, in 2007. She is now a professor at Faculted'Electronique et d'Informatique of USTHB University. Her research interests include remote sensing and image processing. Currently, she is interested in texture analysis and synthesis, remote sensing image retrieval, and three-dimensional (3-D) terrain modelling.

AichoucheBelhadj-Aissa obtained her engineering degree in electronics from National Polytechnic School, Algiers, the magister degree and the Doctorate in image processing and remote sensing from the University of Sciences and Technology Houari Boumediene, Algiers. Currently, she is university Professor and head of the research team "GIS and integration of geo-referenced data". The main research themes focus on modeling and analysis of textures and forms, fusion and classification of objects, SAR interferometry-polarimetry and GIS.
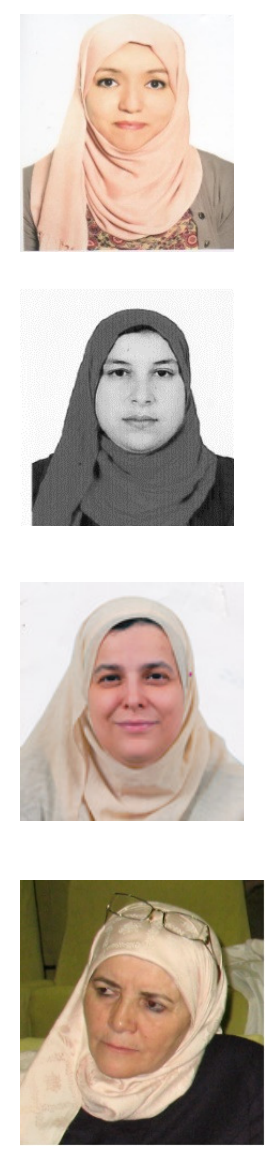